\title{
Logarithmic Newman-Penrose constants for arbitrary polyhomogeneous spacetimes
}

\author{
Juan Antonio Valiente Kroon* \\ School of Mathematical Sciences, \\ Queen Mary \& Westfield College, \\ Mile End Road, London E1 4NS.
}

August 10, 2018

\begin{abstract}
A discussion of how to calculate asymptotic expansions for polyhomogeneous spacetimes using the Newman-Penrose formalism is made. The existence of logarithmic NewmanPenrose constants for a general polyhomogeneous spacetime (i.e. a polyhomogeneous spacetime such that $\left.\Psi_{0}=\mathrm{O}\left(r^{-3} \ln ^{N_{3}}\right)\right)$ is addressed. It is found that these constants exist for the generic case.
\end{abstract}

\section{Introduction}

The study of the gravitational field of isolated sources close to null infinity $\mathscr{I}$ has been done by solving the asymptotic characteristic initial value problem using formal series expansions in powers of $1 / r$, where the coordinate $r$ is either the affine parameter of a future oriented null geodesic or a cleverly constructed luminosity parameter. However, as was noted by some of the first researchers in the area [11], combinations of powers of $\ln r$ and $1 / r$ tend to arise naturally, even if we assume initially expansions in $1 / r$. For long it was thought that these logarithmic terms were in some way connected to the presence of incoming radiation. In order to avoid the appearance of these logarithmic terms ad hoc, some conditions were imposed on the Ansatz for the asymptotic expansions. One of these conditions, used in the work by Bondi et al. [6], [7] and Sachs [23] (Bondi-Sachs (BS) framework) precluded the appearance of $1 / r^{2}$ terms in some of the metric functions. This condition has been known for a long time as the outgoing radiation condition for the gravitational field (ORC), because it bears some resemblance to Sommerfeld's outgoing condition for the wave equation in flat space. It was later realized that the ORC does not preclude the existence of incoming radiation. Moreover, the presence of incoming radiation in itself is not a problem. There are some examples of spacetimes that contain a mixture of incoming and outgoing radiation and that have a somooth $\mathscr{I}$. In any case, what has to be avoided (and is implicit in the definition of an isolated body) is the presence of radiation travelling "infinitely long" distances (i.e. coming from past null infinity $\mathscr{I}^{-}$). But this is achieved by imposing some boundary conditions directly on $\mathscr{I}^{-}[14$.

Newman and Penrose, working with their spin coefficients formalism (NP formalism), used a different condition. Namely, they assumed the $\Psi_{0}$ component of the Weyl tensor to behave asymptotically as $\mathrm{O}\left(r^{-5}\right)$. This condition has its roots in the conformal compactification techniques introduced by Penrose. If one assumes $\Psi_{0}=\mathrm{O}\left(r^{-5}\right)$ then one readily deduces the Peeling theorem, and all conformally rescaled quantities (the spin coefficients, the $\Psi$ 's and the tetrad functions) are well defined at $\mathscr{I}$. But if a slower decay for $\Psi_{0}$ is assumed — say $\mathrm{O}\left(r^{-4}\right)$ - then logarithmic terms will appear again in the asymptotic expansions. One also finds that some conformally rescaled quantities diverge at $\mathscr{I}$. Hence one ends up with a null infinity that is non-smooth.

*E-mail address: j.a.valiente@qmw.ac.uk 
Not surprisingly, the ORC and Penrose's condition are found to be related. If the ORC does not holds (i.e. if we retain the "nasty" $1 / r^{2}$ terms in our initial expansions) then $\Psi_{0}=\mathrm{O}\left(r^{-4}\right)$ [27.

More recently, the work on the non-linear stability of Minkowski spacetime by Christodoulou and Klainerman has shown that generic spacetimes may exhibit a slower fall off (in some cases as low as $\mathrm{O}\left(r^{-7 / 2}\right)$ on $\Psi_{0}$ ) and a non-smooth $\mathscr{I}$. These results together with the fact that logarithmic terms have been observed by other authors in the context of cylindrically symmetric spacetimes [1] and infinite rotating discs [5] suggest that asymptotic expansions just in terms of powers of $1 / r$ only are too restrictive for many physically interesting applications.

From the previous discussion, one can see that a natural way to generalize (and hopefully gain more insight and better understanding!) the work that so far has been done on the asymptotic behaviour of the gravitational field is first, to assume a slower decay for the leading terms of the components of the Weyl tensor, and second, to allow the full appearance of logarithmic terms in the asymptotic expansions 1 . Spacetimes that can be expanded asymptotically in terms of a combination of powers of $1 / r$ and $\ln r$ are known as polyhomogeneous spacetimes.

An analysis of the solutions of the constraint equations by Andersson \& Chruściel [2], [3] seems also to indicate that an adequate framework to study the asymptotic gravitational field is that of the polyhomogeneous spacetimes. Chruściel, MacCallum and Singleton [10] have shown that the hypothesis of polyhomogeneity is formally consistent with the Einstein field equations. Many results that hold in the non-polyhomogeneous setting can be recovered. The BMS group is still the asymptotic symmetry group, and the uniqueness results for the Bondi mass of Chruściel, Jezierski and MacCallum hold [9]. However, the well posedness and existence/uniqueness of the asymptotic characteristic initial value problem is still an open question. Other classical results - like the Peeling theorem - no longer hold, but an adequate generalization can be found in order to encompass the "new" logarithmic terms $[10]$.

Among this last group of results one has that of the Newman-Penrose constants, a set of 10 absolutely conserved quantities that was found to exist for spacetimes satisfying the $\Psi_{0}=\mathrm{O}\left(r^{-5}\right)$ condition. As shown in reference [10] in a particular example, the Newman-Penrose constants are no longer constants for polyhomogeneous spacetimes. However, it was possible to show that for a restricted class of polyhomogeneous spacetimes (those spacetimes with a finite conformally rescaled shear $\widehat{\sigma}$ at $\mathscr{I}$ ) it is possible to construct another set of 10 quantities (that we will call logarithmic Newman-Penrose constants) with a structure similar to that of the NP constants and that are conserved [26].

The objective of this article is to extend the work of references [10] and [26] on these logarithmic constants, i.e. to find a family of functionals of $\Psi_{0}$ that for an arbitrary polyhomogeneous spacetime yields conserved quantities, and for a non-polyhomogeneous spacetime reduces to the NP constants. In order to do so, it will be necessary to study in some detail the polyhomogeneous expansions of a spacetime. Some acquaintance with the NP spin coefficients formalism will be assumed in order to follow the calculations.

Much has been discussed about the interpretation and physical meaning of the NP constants: they have been regarded by some authors as the gravitational analogue of the electric charge, as they retain their values even after a process of radiation [16], [18. However, the works trying to find an interpretation for them have been so far inconclusive, and some authors have even suggested on some grounds that the constants may be devoid of physical meaning [4, 22], [12]. The new set of logarithmic constants adds to this old debate. Once their existence has been shown, the next natural step is to try to attach to them a physical meaning (if any!). In order to do a connection with previous work, stationary polyhomogeneous spacetimes are studied, and their logarithmic NP constants calculated. The result shows that for stationary spacetimes the logarithmic constants have a "logarithmic quadrupole" structure.

This article is organized as follows: in section 2 we give a brief description of the notations and conventions that are used in the present aerticle. In section 3 there is a discussion on how to calculate asymptotic expansion for polyhomogeneous spacetimes using the Newman-Penrose formalism. A description of the tetrad choice is also given. In section 4 we address the problem

\footnotetext{
${ }^{1} \mathrm{~A}$ precision should be done here. We will exclude from our study those spacetimes that contain infinite series in powers of $\ln (r)$.
} 
of how to generalize the Newman-Penrose constants to polyhomogeneous spacetimes (logarithmic NP constants). A detailed study of the evolution equation for the $\Psi_{0}$ component of the Weyl tensor is done. Using this analysis, the main result of this article is proven: the existence of the so called logarithmic Newman-Penrose constants for all polyhomogeneous spacetimes. Finally, there is one appendix. In it one finds 2 tables that summarize the behaviour of generic polyhomogeneous spacetimes. The results listed in these tables are used extensively in most of the arguments in this paper.

\section{Notation \& conventions.}

We will be working with spacetimes that formally can be expanded in terms of powers of $1 / r$ and $\ln r$. For example

$$
\sigma=\sum_{k} \sigma_{k} r^{-k}=\sum_{k} \sum_{j}^{N_{k}} \sigma_{k j} r^{-k} \ln ^{j} r,
$$

where $\sigma_{k}$ denotes the polynomial in $z=\ln r$ with coefficients depending on $u, \theta$ and $\varphi$ associated with $r^{-k}$. A second subscript will mean the power of $z$ to which this function is attached. So, $\sigma_{i, j}$ is the coefficient that comes with $r^{-i} \ln ^{j} r$. For the components of the Weyl tensor superscripts rather than subscripts will be used. Let the symbol \# denote the degree of a polynomial in $z$. We will stick to the convention that the zero polynomial has degree $-\infty$. Hence

$$
\begin{gathered}
\# c(u, \theta, \varphi)=0, \\
\# 0=-\infty .
\end{gathered}
$$

A number in square brackets to the right of a polynomial will also indicate its degree. For instance: $\sigma_{2}\left[N_{3}+1\right]$ means that $\# \sigma_{2}=N_{3}+1$.

We will refer to the NP "field equations", the Bianchi identities and the frame equations in the way they are listed in Stewart's book [24]. In many ocasions one will only be interested in the terms that go with a determinate power of $1 / r$. This will be indicated by attaching a subindex to the name of the equation. A second subindex will refer to a particular power of $\ln r$. So, for instance $(\mathrm{Bb})_{7, N_{6}}$ will mean that we are looking at the relationship that goes with the $r^{7} \ln ^{N_{6}}$ term of the Bianchi identity Bb.

When calculating the expansions of the diverse quantities, one usually needs to evaluate the derivatives and products of polyhomogeneous functions. The following formulae are most useful in these circumstances:

$$
\begin{gathered}
h g=\sum_{i=2} \sum_{k=1}^{i-1} h_{k}(z) g_{i-k}(z) r^{-i}, \\
\frac{\partial}{\partial r} h=\sum_{i=2}\left(h_{i-1}^{\prime}(z)-(i-1) h_{i-1}(z)\right) r^{-i},
\end{gathered}
$$

where $h$ and $g$ are two arbitrary polyhomogeneous functions, the coefficients $h_{k}, g_{k}$ are polynomials in $z=\ln r$, and ' denotes differentiation with respect to $z$.

Some use of the spin weighted spherical harmonics $\left({ }_{s} Y_{l m}\right)$ and the $\widetilde{\delta}$ and $\bar{\varnothing}$ (eth and ethbar) operators will be made. In this case, we will also stick to Stewart's convention that is the same as the one in Penrose and Rindler [21]. Their connection with the NP directional derivatives is

$$
\begin{aligned}
& \check{\partial}=\delta+s(\bar{\alpha}-\beta), \\
& \bar{\gamma}=\bar{\delta}-s(\alpha-\bar{\beta}) .
\end{aligned}
$$




\section{Solving the NP equations using polyhomogeneous expan- sions.}

The first step into solving the NP field equations is to construct a coordinate system and a null tetrad adapted to the problem. Suppose there is a one parameter family of null hypersurfaces. Denote this family by $\mathcal{N}_{u}$. We can use the parameter $u$ to define a scalar field. Of course, this parametrization is completely arbitrary, and one could have used another parameter $\bar{u}$ as long as some smoothness requirements are satisfied. On each of the geodesic generators $\gamma_{u}$ of the null hypersurfaces one can choose an affine parameter $r$. The null hypersurfaces $\mathcal{N}_{u}$ intersect $\mathscr{I}$ in cuts $\mathcal{S}_{u}$. On any one of the cuts, say $\mathcal{S}_{0}$ we can choose arbitrary coordinates $x^{\alpha}, \alpha=2,3$. We can propagate the coordinates $x^{\alpha}$ on $\mathscr{I}$ by demanding $x^{\alpha}=$ const. on each generator of $\mathscr{I}$. In this way we have constructed a coordinate system $\left(u, r, x^{\alpha}\right)$ in the neighborhood of $\mathscr{I}$. There is some freedom left in this construction:

1. we can always choose a different parameter $\bar{u}$ for the family of null hypersurfaces;

2. we can choose a different selection of coordinates $x^{\alpha}$ on $\mathcal{S}_{0}, \bar{x}^{\alpha}=\bar{x}^{\alpha}\left(x^{\alpha}\right)$;

3. we can reset the origin and scaling of the affine parameter $r, \bar{r}=a\left(u, x^{\alpha}\right) r+b\left(u, x^{\alpha}\right)$.

Once we have set our coordinate system, we can construct a null tetrad. An obvious choice for the first element of the tetrad is the gradient of the null hypersurfaces

$$
\mathrm{l}=\mathrm{d} u .
$$

The vector field $l^{\mu}$ is tangent to the geodesic generators $\gamma_{u}$ of $\mathcal{N}_{u}$. We can use the freedom of the scaling of the affine parameter of these generators to set

$$
l^{\mu}=\frac{\partial}{\partial r}
$$

The freedom of the choice of the origin of the affine parameter will be used so that $\rho$ will not contain any $1 / r^{2}$ terms (see equation (26)). Let us denote the 2 -surfaces $u=$ const., $r=$ const. by $\mathcal{S}_{u, r}$. It can be seen that $l^{\mu}$ is future pointing and orthogonal to $\mathcal{S}_{u, r}$. There is only one other null direction with the same properties. The second vector of our tetrad $n^{\mu}$ is chosen to be parallel to this direction. The two other vectors of the tetrad, $m^{\mu}$ and $\bar{m}^{\mu}$, are constructed so that they span the tangent space to $\mathcal{S}_{u, r}, \mathrm{~T}\left(\mathcal{S}_{u, r}\right)$. So from this construction one deduces

$$
\begin{gathered}
n^{\mu}=\frac{\partial}{\partial u}+Q \frac{\partial}{\partial r}+C^{\alpha} \frac{\partial}{\partial x^{\alpha}}, \\
m^{\mu}=\xi^{\alpha} \frac{\partial}{\partial x^{\alpha}},
\end{gathered}
$$

so that the contravariant metric tensor is

$$
g_{N P}^{\mu \nu}=\left(\begin{array}{cccc}
0 & 1 & 0 & 0 \\
1 & 2 Q & C^{\theta} & C^{\varphi} \\
0 & C^{\theta} & -2 \xi^{\theta} \bar{\xi}^{\theta} & -\xi^{\theta} \bar{\xi}^{\varphi}-\bar{\xi}^{\theta} \xi^{\varphi} \\
0 & C^{\varphi} & -\xi^{\theta} \bar{\xi}^{\varphi}-\bar{\xi}^{\theta} \xi^{\varphi} & -2 \xi^{\varphi} \bar{\xi}^{\varphi}
\end{array}\right)
$$

Using a spin-boost it is possible to set $\epsilon=\bar{\epsilon}$ everywhere. From the commutator equations we deduce

$$
\begin{gathered}
\kappa=\epsilon=0, \\
\tau=\bar{\pi}=\bar{\alpha}+\beta
\end{gathered}
$$

and $\rho$ and $\mu$ are real. 
The asymptotic characteristic initial value problem is usually set by supplying $\Psi_{0}$ on an initial null hypersurface $\mathcal{N}_{0}, \sigma$ on $\mathscr{I}^{+}$, and $\Psi_{1}, \operatorname{Re} \Psi_{2}$ and $\xi^{i}$ on $\mathcal{Z}=\mathscr{I}^{+} \cap \mathcal{N}_{0}$ [13].

The most general polyhomogeneous form for $\Psi_{0}$ that is physically reasonable to start with is

$$
\Psi_{0}=\sum_{k=1} \Psi_{0}^{k},
$$

where the coefficients $\Psi_{0}^{k}$ are polynomials in $\ln r$, as mentioned before. With this initial data, the first two equations in the NP hierarchy to be solved are

$$
\begin{gathered}
D \rho=\rho^{2}+\sigma \bar{\sigma}, \\
D \sigma=2 \rho \sigma+\Psi_{0} .
\end{gathered}
$$

Using (41) and (5) into (16) and (17) one obtains the following recurrence relations

$$
\begin{gathered}
\rho_{i-1}^{\prime}-(i-1) \rho_{i-1}=\sum_{k=1}^{i-1}\left(\rho_{k} \rho_{i-k}+\sigma_{k} \bar{\sigma}_{i-k}\right), \\
\sigma_{i-1}^{\prime}-(i-1) \sigma_{i-1}=2 \sum_{k=1}^{i-1} \rho_{k} \sigma_{i-k}+\Psi_{0}^{i} .
\end{gathered}
$$

Setting $i=1$ we find that

$$
\Psi_{0}^{1}=0 .
$$

For $i=2$, the recurrence relations reduce to

$$
\begin{array}{r}
\rho_{1}^{\prime}-\rho_{1}=\rho_{1}^{2}+\sigma_{1} \bar{\sigma}_{1}, \\
\sigma_{1}^{\prime}-\sigma_{1}=2 \rho_{1} \sigma_{1}+\Psi_{0}^{2} .
\end{array}
$$

From equation (21) we see that $\rho_{1}$ has to be a polynomial of degree zero. Couch \& Torrence [8] have shown that in order to have a spacetime with a Weyl tensor that vanishes at infinity then we require $\Psi_{0}=\mathrm{O}\left(r^{-2-\epsilon_{1}}\right)$ where $\epsilon_{1}>0$. Hence, in order to have a physically realistic situation we set

$$
\Psi_{0}^{2}=0,
$$

so that $\sigma_{1}$ is also of degree zero. The remaining system then reduces to

$$
\begin{array}{r}
-\rho_{1}=\rho_{1}^{2}+\sigma_{1} \bar{\sigma}_{1}, \\
-\sigma_{1}=2 \rho_{1} \sigma_{1} .
\end{array}
$$

which has 3 solutions: $\rho_{1}=0, \sigma_{1}=0$ (asymptotically plane) which yields degenerate spacetimes for which $m^{\mu}=\bar{m}^{\mu}=0 ; \rho_{1}=-\frac{1}{2}$ and $\left|\sigma_{1}\right|^{2}=\frac{1}{4}$ (asymptotically cylindrical) which also produces degenerate spacetimes for which $m^{\mu}=\bar{m}^{\mu}$; and $\rho_{1}=-1, \sigma_{1}=0$ which gives rise to spacetimes that are asymptotically minkowskian. In what follows we will stick to this last solution.

For $i=3$ one finds that

$$
\begin{aligned}
\rho_{2}^{\prime} & =0, \\
\sigma_{2}^{\prime} & =\Psi_{0}^{3} .
\end{aligned}
$$

Therefore $\rho_{2}$ is a polynomial of degree 0 in $\ln r$. Using the remaining freedom in the definition of the radial coordinate (recall that $r$ is an affine parameter) it is possible to redefine $r$ so that $\rho_{2}=0$ (see for example [19]). From equation (27) one reads

$$
\Psi_{0}^{3, j}=(j+1) \sigma_{2, j+1},
$$

$j=0 \ldots N_{3}$, whence $\# \sigma_{2}=N_{3}+1$. So, the only part of $\sigma_{2}$ that is not determined by the data on the initial hypersurface $\mathcal{N}$ is $\sigma_{2,0}$, in complete agreement with the results on the well posedness of the asymptotic characteristic initial value problem. We can think of $\Psi_{0}^{3, j} j=0 \ldots N-1$ as 
a contribution to the shear from the incoming radiation using Szekeres interpretation of the components of the Weyl tensor ( $\Psi_{0}$ can be regarded as an incoming transverse wave) [25].

For $i=4$ one obtains,

$$
\begin{array}{r}
\rho_{3}^{\prime}-\rho_{3}=\sigma_{2} \bar{\sigma}_{2}, \\
\sigma_{3}^{\prime}-\sigma_{3}=\Psi_{0}^{4} .
\end{array}
$$

From these two equations one can readily see that $\# \rho_{3}=2 N_{3}+2$ and $\# \sigma_{3}=N_{4}$. Recurrence relations to calculate the coefficients of the two polynomials can be easily obtained.

The process described here can be performed up to any desired order in $1 / r$. A similar analysis can be done with the remaining field equations, Bianchi identities and commutator relations. The order of solving the equations is given in reference [24. A summary of this analysis is presented in the form of tables in Appendix A.

\section{Logarithmic NP constants for a generic polyhomogeneous spacetime.}

We are interested in the construction of conserved quantities that can be expressed as integrals over a cut of $\mathscr{I}, S^{2}$. One expects these constants to be formed out of quantities that are initial data on the initial null hypersurface $\mathcal{N}_{0}$. As $\Psi_{1}^{4,0}, \operatorname{Re} \Psi_{2}^{3,0}$, and $\sigma_{2,0}$ (the data that has to be prescribed on $\mathscr{I}+\cap \mathcal{N}_{0}$ ) are used to construct the Bondi mass and the angular momentum of the spacetime - quantities that are not conserved-, we do not expect to be able to construct conserved quantities out of them.

Newman \& Penrose [16], 18] have found that for non-polyhomogeneous spacetimes, 10 conserved quantities can be constructed out of $\Psi_{0}$. As it has been mentioned before, these quantities are not conserved for polyhomogeneous spacetimes. Nevertheless, in the case when the leading term of the shear $\left(\sigma_{2}\right)$ contains no $\ln r$ terms (which corresponds to the situation $\# \Psi_{0}^{3}=-\infty$ ) it was possible to single out some other quantities with a similar structure (logarithmic NP constants) that are conserved. We want now to show that even for the most general class of polyhomogeneous spacetimes it is possible to construct such constants.

The key idea in the construction of these constants is to deduce from the Bianchi identities an equation whose left hand side is a derivative with respect to the retarded time $u$ of one of the coefficients of $\Psi_{0}$, and whose right hand side is an $\bar{\partial}$ derivative of some combination of the coefficients in $\Psi_{0}, \Psi_{1}, \Psi_{2}$; that is, equations of the form

$$
\dot{\Psi}_{0}^{i, M}=\bar{\varnothing}(F),
$$

where ${ }^{\cdot}$ denotes differentiation with respect to $u$, and the indices $i$ and $M$ are to be determined. Note that this equation has the same structure as the continuity equations in mechanics of continuous media: a derivative with respect to time plus the divergence of a flux, $F$ 2. Equation (31) has an overall spin weight 2 , and the function $F$ spin weight 3.

We multiply equation (31) by $\left({ }_{2} \bar{Y}_{l m}\right)$, a spin 2 weighted spherical harmonic, in order to obtain an equation of spin weight 0 . Now, we integrate over the unit sphere (a cut of $\mathscr{I}$ ) so that

$$
\frac{\partial}{\partial u}\left(\int_{S^{2}} \Psi_{0}^{i, M}\left({ }_{2} \bar{Y}_{l m}\right) \mathrm{d} S\right)=\int_{S^{2}} \bar{\delta}(F)\left({ }_{2} \bar{Y}_{l m}\right) \mathrm{d} S .
$$

Using the identity [16]

$$
\int_{S^{2}}\left({ }_{s} \bar{Y}_{l, m}\right) \overline{\bar{\delta}}^{l-s+1} \zeta \mathrm{d} S=0
$$

where $\zeta$ is of spin weight $l+1$ (in our case $\zeta=F$ has spin weight 3 , hence $l=2$ ), we end up with

\footnotetext{
${ }^{2}$ The operator $\bar{\partial} \partial$ applied to a quantity of spin weight zero is equal to the angular part of the laplacian, $\nabla^{2}$. Hence, we can regard $\bar{\partial} \check{\partial}$ as the laplacian for spin weighted quantities, and $\precsim$ or $\bar{\varnothing}$ as divergences ("square root of the laplacian").
} 


$$
\frac{\partial}{\partial u}\left(\int_{S^{2}} \Psi_{0}^{i, M}\left(_{2} \bar{Y}_{2 m}\right) \mathrm{d} S\right)=0 .
$$

Hence the 5 complex quantities

$$
\mathcal{Q}^{i, M}=\int_{S^{2}} \Psi_{0}^{i, M}\left({ }_{2} \bar{Y}_{2 m}\right) \mathrm{d} S,
$$

$m=-2,-1 \ldots 2$ are conserved as long as equation (31) holds.

The results in 26] show that one should expect to find the desired conservation laws in the coefficients of the highest power of $\ln r$ in the $1 / r^{6}$ terms of the evolution equation for $\Psi_{0}$. An algebraic explanation of this fact can be found by looking at the identity

$$
\frac{\partial}{\partial r}\left(A(u, \theta, \varphi) r^{-i} \ln ^{j} r\right)=(-i) A(u, \theta, \varphi) r^{-i-1} \ln ^{j} r+j A(u, \theta, \varphi) r^{-i-1} \ln ^{j-1} r,
$$

Hence, differentiation with respect to $r$ adds extra terms to the lower powers of $\ln r$, but not to the highest. Therefore, the coefficients of the highest power of $\ln r$ (for a given power of $1 / r$ ) mimic the behaviour of the coefficients in a non-polyhomogeneous spacetime. The evolution equations in both cases will have the same algebraic form.

The situation discussed in this article is more elaborate than the one described in [26] as one has to deal with many new terms coming from $\Psi_{0}^{3}$. It will be necessary to keep track of the behaviour of the degrees $\left(N_{i}\right)$ of the polynomials $\Psi_{0}^{i}$ as the system evolves. Therefore our discussion will start at the lowest order $\left(1 / r^{3}\right)$ instead of just going directly to the $1 / r^{6}$ order terms.

As discussed previously, the most general form for $\Psi_{0}$ is the following:

$$
\Psi_{0}=\Psi_{0}^{3}\left[N_{3}\right] r^{-3}+\Psi_{0}^{4}\left[N_{4}\right] r^{-4}+\Psi_{0}^{5}\left[N_{5}\right] r^{-5}+\Psi_{0}^{6}\left[N_{6}\right] r^{-6}+\cdots,
$$

and its evolution equation is the Bianchi identity $(\mathrm{Bb})$,

$$
\Delta \Psi_{0}-\delta \Psi_{1}=(4 \gamma-\mu) \Psi_{0}-2(2 \tau+\beta) \Psi_{1}+3 \sigma \Psi_{2} .
$$

One expects the values of $N_{i}$ to change as the system evolves. So, we can think of these numbers as discrete functions of the retarded time $u\left(N_{i}=N_{i}(u)\right)$. A tilde $\sim$ over one of these numbers will mean that we are referring to its value at the initial null hypersurface $\mathcal{N}_{0}$.

The equation $(\mathrm{Bb})_{3}$ readily yields

$$
\dot{\Psi}_{0}^{3}=0
$$

whence the polynomial $\Psi_{0}^{3}$ does not depend on $u$, and its degree $\left(\# \Psi_{0}^{3}=\widetilde{N}_{3}=N_{3}\right)$ is preserved during evolution.

From $(\mathrm{Bb})_{4}$ we obtain

$$
\dot{\Psi}_{0}^{4}-\frac{1}{2} \Psi_{0}^{3 \prime}\left[N_{3}-1\right]+\Psi_{0}^{3}\left[N_{3}\right]-\partial \Psi_{1}^{3}\left[N_{3}\right]=0,
$$

an equation of the form

$$
\dot{\Psi}_{0}^{4}=P\left[N_{3}\right],
$$

$P\left[N_{3}\right]$ a polynomial of degree $N_{3}$. If at the initial null hypersurface $\mathcal{N}_{0}$ we have $\tilde{N}_{4}>N_{3}$ then we find that

$$
\dot{\Psi}_{0}^{4, N_{4}}=\dot{\Psi}_{0}^{4, N_{4}-1}=\cdots=\dot{\Psi}_{0}^{4, N_{3}+1}=0,
$$

hence $\Psi_{0}^{4, N_{4}}, \ldots, \Psi_{0}^{4, N_{3}+1}$ will be constants of motion, and the number $N_{4}$ a constant. The first evolution equation where the interaction between $r^{-3}$ and $r^{-4}$ terms shows is

$$
\dot{\Psi}_{0}^{4, N_{3}}+\Psi_{0}^{3, N_{3}}-\partial \Psi_{1}^{3, N_{3}}=0 .
$$

Using, $(\mathrm{Ba})_{4}$

$$
\Psi_{1}^{3 \prime}\left[N_{3}-1\right]+\Psi_{1}^{3}\left[N_{3}\right]=\bar{\partial} \Psi_{0}^{3}\left[N_{3}\right],
$$


and the commutator for the derivatives we obtain

$$
\dot{\Psi}_{0}^{4, N_{3}}+3 \Psi_{0}^{3, N_{3}}=\bar{\partial} \partial \Psi_{0}^{3, N_{3}},
$$

so that we cannot derive a conservation law from this equation due to the presence of the extra $3 \Psi_{0}^{3}$ term. If $N_{3} \geq \widetilde{N}_{4}$ then the only thing we can affirm is that $N_{3} \geq N_{4}$ (from (41)).

The $(\mathrm{Bb})_{5}$ equation is much more involved.

$$
\begin{array}{r}
\dot{\Psi}_{0}^{5}\left[N_{5}\right]-\frac{1}{2}\left(\Psi_{0}^{4 \prime}-4 \Psi_{0}^{4}\right)+Q_{1}\left[N_{3}+1\right]\left(\Psi_{0}^{3 \prime}-3 \Psi_{0}^{3}\right)\left[N_{3}\right]+C_{2}^{\alpha}\left[N_{3}+1\right] \partial_{\alpha} \Psi_{0}^{3}\left[N_{3}\right] \\
-\delta_{1} \Psi_{1}^{4}[Y]+\delta_{2}\left[N_{3}+1\right] \Psi_{1}^{3}\left[N_{3}\right]= \\
4 \gamma_{2}\left[N_{3}+1\right] \Psi_{0}^{3}\left[N_{3}\right]+\frac{1}{2} \Psi_{0}^{4}\left[N_{4}\right]-\mu_{2}\left[N_{3}+1\right] \Psi_{0}^{3}\left[N_{3}\right]-4 \tau_{2}\left[N_{3}+1\right] \Psi_{1}^{3}\left[N_{3}\right] \\
-2 \beta_{1} \Psi_{1}^{4}[Y]-2 \beta_{2}\left[N_{3}+1\right] \Psi_{1}^{3}\left[N_{3}\right]+3 \sigma_{2}\left[N_{3}+1\right] \Psi_{2}^{3}\left[N_{3}+1\right]
\end{array}
$$

an equation of the form

$$
\dot{\Psi}_{0}^{5}=P[Y]
$$

where $Y=\max \left\{N_{4}+1,2 N_{3}+2\right\}$. Note that if $\widetilde{N}_{4}+1>2 N_{3}+2$ then $\widetilde{N}_{4}>N_{3}$, and again due to $(\mathrm{Bb})_{4}, N_{4}$ will be a constant. Hence in this case $Y$ is a constant. If in the other hand, $\widetilde{N}_{4}+1 \leq 2 N_{3}+2$ we end up with two cases $\left(N_{3}<\widetilde{N}_{4}\right.$ or $\left.N_{3} \geq \widetilde{N}_{4}\right)$. If $N_{3}<\widetilde{N}_{4}$ then $N_{4}$ is a constant, and then the inequality $2 N_{3}+2 \geq N_{4}+1$ holds for later times because it held at $\mathcal{N}_{0}$. And if $N_{3} \geq \widetilde{N}_{4}$ then $N_{3} \geq N_{4}$, and hence again $2 N_{3}+2 \geq N_{4}+1$. The conclusion is that $Y$ is a constant again.

Looking at equation (46) we find that if $\widetilde{N}_{5}>\max \left\{N_{4}+1,2 N_{3}+2\right\}$ we obtain a set of constants of motion $\left(\Psi_{0}^{5, N_{5}}, \ldots, \Psi_{0}^{5, Y+1}\right)$. If $\widetilde{N_{5}} \leq Y=\max \left\{N_{4}+1,2 N_{3}+2\right\}$ at $\mathcal{N}_{0}$ then we will not have constants of motion. The first equation showing an interaction between terms of different orders in $r$ is equation $(\mathrm{Bb})_{5, Y}$ :

$$
\dot{\Psi}_{0}^{5, Y}=\partial \Psi_{1}^{4, Y}+\left\{\sigma_{2, N_{3}+1} \Psi_{2}^{3, N_{3}+1}\right\}
$$

where the term in curly brackets \{\} is present if $Y=2 N_{3}+2$. In order to obtain an equation in terms of "initial data" quantities only we require the equations $(\mathrm{Ba})_{5}$ and $(\mathrm{Bc})_{4}$ :

$$
\begin{gathered}
\Psi_{1}^{4 \prime}\left[N_{4}\right]=\bar{\partial} \Psi_{0}^{4}\left[N_{4}\right]+\left(\bar{\delta}^{2}-4 \alpha_{2}\right)\left[N_{3}+1\right] \Psi_{0}^{3}\left[N_{3}\right]+\pi_{2}\left[N_{3}+1\right] \Psi_{0}^{3}\left[N_{3}\right] . \\
\Psi_{2}^{3 \prime}\left[N_{3}\right]-\bar{\partial} \Psi_{1}^{3}\left[N_{3}\right]=-\lambda_{1}[0] \Psi_{0}^{3}\left[N_{3}\right]
\end{gathered}
$$

Using equations (49) and (59) it is not hard to show that equation (48) does not have the required form.

Following [18] and 226] we expect to find conserved quantities of the desired form (if any) in the $(\mathrm{Bb})_{6}$ terms. The equation in this case is extremely involved, so it is better to look at it in parts:

$$
\begin{aligned}
{\left[\dot{\Psi}_{0}\right]_{6}=} & \dot{\Psi}_{0}^{6}\left[N_{6}\right], \\
{\left[Q \partial_{r} \Psi_{0}\right]_{6}=} & -\frac{1}{2}\left(\Psi_{0}^{5 \prime}-5 \Psi_{0}^{5}\right)\left[N_{5}\right]+Q_{1}\left[N_{3}+1\right]\left(\Psi_{0}^{4 \prime}-4 \Psi_{0}^{4}\right)\left[N_{4}\right] \\
& +Q_{2}[Y]\left(\Psi_{0}^{3 \prime}-3 \Psi_{0}^{3}\right)\left[N_{3}\right] \\
{\left[C^{\alpha} \partial_{\alpha} \Psi_{0}\right]_{6}=} & C_{2}^{\alpha}\left[N_{3}+1\right] \partial_{\alpha} \Psi_{0}^{4}\left[N_{4}\right]+C_{3}^{\alpha}[Y] \partial_{\alpha} \Psi_{0}^{3}\left[N_{3}\right], \\
{\left[\delta \Psi_{1}\right]_{6}=} & \delta_{1} \Psi_{1}^{5}\left[N_{5}\right]+\delta_{2}\left[N_{3}+1\right] \Psi_{1}^{4}[Y] \\
& +\delta_{3}\left[\max \left\{N_{4}, 2 N_{3}+2\right\}\right] \Psi_{1}^{3}\left[N_{3}\right] \\
{\left[\gamma \Psi_{0}\right]_{6}=} & \gamma_{2}\left[N_{3}+1\right] \Psi_{0}^{4}\left[N_{4}\right]+\gamma_{3}[Y] \Psi_{0}^{3}\left[N_{3}\right] \\
{\left[\mu \Psi_{0}\right]_{6}=} & -\frac{1}{2} \Psi_{0}^{5}\left[N_{5}\right]+\mu_{2}\left[N_{3}+1\right] \Psi_{0}^{4}\left[N_{4}\right]+\mu_{3}[Y] \Psi_{0}^{3}\left[N_{3}\right], \\
{\left[\tau \Psi_{1}\right]_{6}=} & \tau_{2}\left[N_{3}+1\right] \Psi_{1}^{4}[Y]+\tau_{3}[Y] \Psi_{1}^{3}\left[N_{3}\right], \\
{\left[\beta \Psi_{1}\right]_{6}=} & \beta_{1} \Psi_{1}^{5}\left[N_{5}\right]+\beta_{2}\left[N_{3}+1\right] \Psi_{1}^{4}[Y]+\beta_{3}[Y] \Psi_{1}^{3}\left[N_{3}\right], \\
{\left[\sigma \Psi_{2}\right]_{6}=} & \sigma_{2}\left[N_{3}+1\right] \Psi_{2}^{4}[Y]+\sigma_{3}\left[N_{4}\right] \Psi_{2}^{3}\left[N_{3}+1\right] .
\end{aligned}
$$


Hence, $(\mathrm{Bb})_{6}$ is an equation of the form

$$
\dot{\Psi}_{0}^{6}=P[X]
$$

where $X=\max \left\{N_{5}, 3 N_{3}+3, N_{3}+N_{4}+2\right\}=\max \left\{N_{5},\left(N_{3}+1\right)+Y\right\}$. Now, if initially $\widetilde{N}_{5}>$ $\left(N_{3}+1\right)+Y$ then $N_{5}>Y$, and using $(\mathrm{Bb})_{5}$ we see that $N_{5}$ is a constant. On the other hand, if $\widetilde{N}_{5} \leq\left(N_{3}+1\right)+Y$ then we end up with two possibilities: $\widetilde{N}_{5}>Y$ which yields $N_{5}$ constant; and $\widetilde{N}_{5} \leq Y$ from which one deduces $N_{5} \leq Y$. This arguments show the remarkable fact that the number

$$
X=\max \left\{N_{5}, 3 N_{3}+3, N_{3}+N_{4}+2\right\}
$$

is a constant independent of the initial values of $N_{3}, N_{4}$ and $N_{5}$.

As in previous orders, we will have a set of constants of motion if $N_{6}>X=\max \left\{N_{5}, 3 N_{3}+\right.$ $\left.3, N_{3}+N_{4}+2\right\}: \Psi_{0}^{6, N_{6}}, \ldots, \Psi_{0}^{6, X+1}$. The first non-trivial evolution equation will be

$$
\begin{array}{r}
\dot{\Psi}_{0}^{6, X}+\left\langle\frac{5}{2} \Psi_{0}^{5, X}\right\rangle+\left\langle\delta_{1} \Psi_{1}^{5, X}\right\rangle+\left\{\delta^{2, N_{3}+1} \Psi_{1}^{4, Y}\right\}= \\
\left\langle\frac{1}{2} \Psi_{0}^{5, X}\right\rangle-\left\{4 \tau_{2, N_{3}+1} \Psi_{1}^{4, Y}\right\}-\left\langle 2 \beta_{1} \Psi_{1}^{5, X}\right\rangle-\left\{2 \beta_{2, N_{3}+1} \Psi_{1}^{4, Y}\right\} \\
+\left\{3 \sigma_{2, N_{3}+1} \Psi_{2}^{4, Y}\right\} .
\end{array}
$$

The terms in curly brackets \{\} will not be present if $X=N_{5}$, while the terms in angle brackets 〈〉 will not appear if $X=\max \left\{3 N_{3}+3, N_{3}+N_{4}+2\right\}$.

In order to obtain an equation that contains only "initial data" quantities we will need to use the equations $(\mathrm{Ba})_{6}$ and $(\mathrm{Bc})_{5}$ :

$$
\begin{gathered}
\Psi_{1}^{5 \prime}\left[N_{5}-1\right]-\Psi_{1}^{5}\left[N_{5}\right]-\bar{\delta} \Psi_{0}^{5}\left[N_{5}\right]-\bar{\delta}^{2}\left[N_{3}+1\right] \Psi_{0}^{3}\left[N_{3}\right] \\
-\bar{\delta}^{3}\left[\max \left\{N_{4}, 2 N_{3}+2\right\}\right] \Psi_{0}^{3}\left[N_{3}\right]= \\
\pi_{2}\left[N_{3}+1\right] \Psi_{0}^{4}\left[N_{4}\right]+\pi_{3}[Y] \Psi_{0}^{3}\left[N_{3}\right]-4 \alpha_{2}\left[N_{3}+1\right] \Psi_{0}^{4}\left[N_{4}\right] \\
-4 \alpha_{3}[Y] \Psi_{0}^{3}\left[N_{3}\right]+4 \rho_{3}\left[2 N_{3}+2\right] \Psi_{0}^{3}\left[N_{3}\right] \\
-\lambda_{1} \Psi_{0}^{4}\left[N_{4}\right]-\lambda_{2}\left[N_{3}+1\right] \Psi_{0}^{3}\left[N_{3}\right]+2 \pi_{2}\left[N_{3}+1\right] \Psi_{1}^{3}\left[N_{3}\right]-2 \alpha_{2}\left[N_{3}+1\right] \Psi_{1}^{3}\left[N_{3}\right] .
\end{gathered}
$$

From equation (63) we obtain (when $X=N_{5}$ )

$$
\Psi_{1}^{5, N_{5}}=-\bar{\partial} \Psi_{0}^{5, N_{5}}
$$

and from (64)

$$
\Psi_{2}^{4, Y}=-\bar{\partial} \Psi_{1}^{4, Y}
$$

Substitution of these results into equation (62) yields

$$
\begin{aligned}
\dot{\Psi}_{0}^{6, X}= & \left\langle\partial \bar{\partial} \Psi_{0}^{5, N_{5}}\right\rangle-\left\langle 2 \Psi_{0}^{5, N_{5}}\right\rangle-\left\{\left(\delta^{2, N_{3}+1}-2 \beta_{2, N_{3}+1}\right) \Psi_{1}^{4, Y}\right\} \\
& -\left\{4 \tau_{2, N_{3}+1} \Psi_{1}^{4, y}\right\}-\left\{3 \sigma_{2, N_{3}+1} \bar{\partial} \Psi_{1}^{4, Y}\right\} .
\end{aligned}
$$

Using the techniques described in the previous section is not difficult (but very lenghty!) to obtain the values of the coefficients $\delta^{2, N_{3}+1}, \beta_{2, N_{3}+1}, \tau_{2, N_{3}+1}$, and $\sigma_{2, N_{3}+1}$ in terms of $\Psi_{0}^{3}$ :

$$
\delta^{2, N_{3}+1}=-\frac{1}{N_{3}+1} \Psi_{0}^{3, N_{3}} \bar{\delta}^{1}
$$




$$
\begin{aligned}
\beta_{2, N_{3}+1} & =\frac{-\alpha_{1}}{N_{3}+1} \Psi_{0}^{N_{3}}, \\
\tau_{2, N_{3}+1} & =\frac{1}{N_{3}+1} \bar{ð} \Psi_{0}^{3, N_{3}}, \\
\sigma_{2, N_{3}+1} & =\frac{1}{N_{3}+1} \Psi_{0}^{3, N_{3}} .
\end{aligned}
$$

With them and the commutator relation

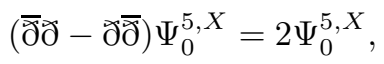

one obtains an equation with the same structure as (31):

$$
\dot{\Psi}_{0}^{6, X}=-\bar{\varnothing}\left(\left\langle\partial \Psi_{0}^{5, X}\right\rangle+\left\{\frac{4}{N_{3}+1} \Psi_{0}^{3, N_{3}} \Psi_{1}^{4, Y}\right\}\right) .
$$

At this point, the relevance of the constancy of $X$ becomes clear. If it were not the case, then the continuity equation (73) could change as the system evolves, and we would have different constants at different null hypersurfaces!

Therefore we have proven the following proposition:

Proposition 1 The quantities (5 complex in general)

$$
\mathcal{Q}_{k}^{X}=\int_{S^{2}} \Psi_{0}^{6, X}\left({ }_{2} \bar{Y}_{2 k}\right) d S
$$

with $X=\max \left\{N_{5}, 3 N_{3}+3, N_{3}+N_{4}+2\right\}$ are constants of motion for any polyhomogeneous spacetime.

Observe that if $\widetilde{N}_{3}=\widetilde{N}_{4}=-\infty$ and $\widetilde{N}_{5}=0$ (that encompasses the non-polyhomogeneous case studied by Newman and Penrose) then $X=0$, and the logarithmic constants reduce to the NP constants. While if $N_{3}=-\infty$ then $X=N_{5}$ and we recover the logarithmic constants of [26]. Hence we have succeded in our primary objective of finding a family of functionals of the quantity $\Psi_{0}$ that are absolutely conserved, and that reduce to the NP constants in the non-polyhomogeneous framework.

\section{Conclusions}

It has been shown that there is a functional of $\Psi_{0}$ that for a polyhomogeneous spacetime yields 10 absolutely conserved quantities, and for a non-polyhomogeneous spacetime reduce to the NP constants.

There are still some remaining open questions. One of them is related with the well known fact that for linear fields one has an infinite hierarchy of NP constants. The general feeling in the case of the NP and logarithmic NP constants for the gravitational field is that the only quantities that one can form that have the desired properties are precisely the ones already known. However a proof is still not available.

The other main question is that of the physical interpretation of the quantities. A step forward in this line would be the evaluation of the constants for some non-stationary spacetime. These topics will be the subject of future work.

\section{Acknowlegments}

I would like to thank Prof M A H MacCallum for his continuous encouragement and advice. I also would like to thank Dr G D Kerr for numerous discussions on spin weighted quantities and the $\partial$ and $\bar{\partial}$, and to Dr R Lazkoz for several suggestions. An important precision by Dr R Beig is also acknowledged. I am supported by the Consejo Nacional de Ciencia y Tecnología (CONACYT), Mexico, grant 110441/110491. 


\section{A Leading terms for the spin coefficients}

The objective of the present appendix is to present in a concise way the results of the calculations described in section 3. The tables give the degrees of the different polynomials that appear in the polyhomogeneous expansions. These tables are indispensable if one wishes to follow the calculations in full detail. As mentioned before, a $-\infty$ means that the given polynomial is zero.

\section{A.1 General polyhomogeneous spacetimes $\left(N_{3} \neq-\infty\right)$.}

\begin{tabular}{|c|c|c|c|c|c|}
\hline & 1 & $r^{-1}$ & $r^{-2}$ & $r^{-3}$ & $r^{-4}$ \\
\hline$\Psi_{0}$ & $-\infty$ & $-\infty$ & $-\infty$ & $N_{3}$ & $\max \left\{N_{4}+1,2 N_{3}+2\right\}$ \\
\hline$\Psi_{1}$ & $-\infty$ & $-\infty$ & $-\infty$ & $N_{3}+1$ & $\max \left\{N_{4}+1,2 N_{3}+2\right\}$ \\
\hline$\Psi_{2}$ & $-\infty$ & $-\infty$ & $-\infty$ & & \\
\hline$\Psi_{3}$ & $-\infty$ & $-\infty$ & 0 & $2 N_{3}+2$ & $N_{3}+N_{4}+1$ \\
\hline$\Psi_{4}$ & $-\infty$ & 0 & 0 & $N_{4}$ & \\
\hline$\rho$ & $-\infty$ & 0 & $-\infty$ & $\max \left\{N_{4}+1,2 N_{3}+2\right\}$ & \\
\hline$\sigma$ & $-\infty$ & $-\infty$ & $N_{3}+1$ & $\max \left\{N_{4}+1,2 N_{3}+2\right\}$ & \\
\hline$\alpha$ & $-\infty$ & 0 & $N_{3}+1$ & $\max \left\{N_{4}+1,2 N_{3}+2\right\}$ & \\
\hline$\beta$ & $-\infty$ & 0 & $N_{3}+1$ & $\max \left\{N_{4}+1,2 N_{3}+2\right\}$ & \\
\hline$\tau$ & $-\infty$ & $-\infty$ & $N_{3}+1$ & $\max \left\{N_{4}+1,2 N_{3}+2\right\}$ & \\
\hline$\pi$ & $-\infty$ & $-\infty$ & $N_{3}+1$ & $\max \left\{N_{4}+1,2 N_{3}+2\right\}$ & \\
\hline$\gamma$ & $-\infty$ & $-\infty$ & $N_{3}+1$ & $\max \left\{N_{4}+1,2 N_{3}+2\right\}$ & \\
\hline$\lambda$ & $-\infty$ & 0 & $N_{3}+1$ & $\max \left\{N_{4}+1,2 N_{3}+2\right\}$ & \\
\hline$\mu$ & $-\infty$ & 0 & $N_{3}+1$ & $\max \left\{N_{4}, 2 N_{3}+2\right\}$ & \\
\hline$\nu$ & $-\infty$ & $-\infty$ & $N_{3}+1$ & $\max \left\{N_{4}+1,2 N_{3}+2\right\}$ & \\
\hline$\xi^{\alpha}$ & $-\infty$ & 0 & $N_{3}+1$ & & \\
\hline$C^{\alpha}$ & $-\infty$ & $-\infty$ & $N_{3}+1$ & & \\
\hline$Q$ & 0 & $N_{3}+1$ & $\max \left\{N_{4}+1,2 N_{3}+2\right\}$ & & \\
\hline
\end{tabular}

\section{A.2 Polyhomogeneous space-times with a finite shear at $\mathscr{I}$.}

\begin{tabular}{|c|c|c|c|c|c|c|c|}
\hline & 1 & $r^{-1}$ & $r^{-2}$ & $r^{-3}$ & $r^{-4}$ & $r^{-5}$ & $r^{-6}$ \\
\hline$\Psi_{0}$ & $-\infty$ & $-\infty$ & $-\infty$ & $-\infty$ & $N_{4}$ & $N_{5}$ & $N_{6}$ \\
\hline$\Psi_{1}$ & $-\infty$ & $-\infty$ & $-\infty$ & $-\infty$ & $N_{4}+1$ & $N_{5}$ & \\
\hline$\Psi_{2}$ & $-\infty$ & $-\infty$ & $-\infty$ & 0 & $N_{4}+1$ & $N_{5}$ & \\
\hline$\Psi_{3}$ & $-\infty$ & $-\infty$ & 0 & 0 & & & \\
\hline$\Psi_{4}$ & $-\infty$ & 0 & 0 & & & & \\
\hline$\rho$ & $-\infty$ & 0 & $-\infty$ & 0 & $N_{4}$ & & \\
\hline$\sigma$ & $-\infty$ & $-\infty$ & 0 & $N_{4}$ & $N_{5}$ & & \\
\hline$\alpha$ & $-\infty$ & 0 & 0 & $N_{4}+1$ & & & \\
\hline$\beta$ & $-\infty$ & 0 & 0 & $N_{4}+1$ & & & \\
\hline$\tau$ & $-\infty$ & $-\infty$ & 0 & $N_{4}+1$ & & & \\
\hline$\pi$ & $-\infty$ & $-\infty$ & 0 & $N_{4}+1$ & & & \\
\hline$\gamma$ & $-\infty$ & $-\infty$ & 0 & $N_{4}+1$ & & & \\
\hline$\lambda$ & $-\infty$ & 0 & 0 & $N_{4}+1$ & & & \\
\hline$\mu$ & $-\infty$ & 0 & 0 & $N_{4}+1$ & & & \\
\hline$\nu$ & $-\infty$ & $-\infty$ & 0 & $N_{4}+1$ & & & \\
\hline$\xi^{\alpha}$ & $-\infty$ & 0 & 0 & $N_{4}$ & & & \\
\hline$C^{\alpha}$ & $-\infty$ & $-\infty$ & 0 & $N_{4}+1$ & & & \\
\hline$Q$ & 0 & 0 & $N_{4}+1$ & & & & \\
\hline
\end{tabular}

\section{References}


[1] A Ashtekar, J Bičák \& B G Schmidt. Behavior of Einstein-Rosen waves at null infinity. Phys. Rev. D 55, 687 (1997).

[2] L Andersson \& P T Chruściel. Hyperboloidal Cauchy data for vacuum Einstein equations and obstructions to the smoothness of null infinity. Phys. Rev. Lett. 70, 2829 (1993).

[3] L Andersson \& P T Chruściel. On 'hyperboloidal' Cauchy data for vacuum Einstein equations and obstructions to the smoothness of $\mathscr{I}$. Comm. Math. Phys. 161533 (1994).

[4] J M Bardeen \& W H Press. Radiation fields in the Schwarzschild background. J. Math. Phys. 14, 7 (1993).

[5] J Bičák, D Lynden-Bell \& C Pichon. Relativistic discs and flat galaxy models. Mon. Not. R. astr. Soc. 265, 126.

[6] H Bondi, M G J van der Burg \& A W K Metzner. Gravitational waves in general relativity VII. Waves from axi-symmetric isolated systems. Proc. R. Soc. Lond. A 269, 21 (1962).

[7] M G J van der Burg. Gravitational waves in general relativity IX. Conserved quantities. Proc. Roy. Soci Lond. A 294, 112 (1966).

[8] W E Couch \& R J Torrence. Asymptotic behaviour of vacuum spacetimes. J. Mat. Phys. 13, 69 (1972).

[9] P T Chruściel, J Jezierski \& M A H MacCallum. Uniqueness of the Trautman-Bondi mass. Phys. Rev. D 58, 084001 (1998).

[10] P T Chruściel, M A H MacCallum \& D B Singleton. Gravitational waves in general relativity $X I V$. Bondi expansions and the "polyhomogeneity" of Scri. Phil. Trans. Roy. Soc. Lond. A. 350, 113 (1995).

[11] V A Fock. Rev. Mod. Phys. 29, 325 (1957).

[12] J N Goldberg. Conservation of the Newman-Penrose conserved quantities. Phys. Rev. Lett. 28, 1400 (1972).

[13] J Kánnár. On the existence of $C^{\infty}$ solutions to the asymptotic characteristic initial value problem in general relativity. Proc. Roy. Soc. Lond. A 452, 945 (1996).

[14] G Leipold \& M Walker. Boundary conditions at past null infinity for zero rest mass fields including gravitation. Ann. Inst. Henri Poincare XXVII, 61 (1977).

[15] E T Newman \& R Penrose.An approach to gravitational radiation by a method of Spin coefficients. J. Math. Phys. 3, 566 (1962).

[16] E T Newman \& R Penrose. 10 exact gravitationally conserved quantities. Phys. Rev. Lett. 15, $231(1965)$.

[17] E T Newman \& R Penrose. Note on the Bondi-Metzner-Sachs Group. J. Math. Phys. 7, 863 (1966).

[18] E T Newman \& R Penrose. New conservation laws for zero rest mass fields in asymptotically flat space-time. Proc. Roy Soc. A. 305, 175 (1968).

[19] E T Newman \& T W J Unti. Behavior of asymptotically flat empty spaces. J. Math. Phys. 3, 891 (1962).

[20] R Penrose. Zero rest-mass fields including gravitation: asymptotic behaviour. proc. Roy. Soc. Lond. A 284, 159 (1965).

[21] R Penrose \& W Rindler. Spinors and spacetime 1: two-spinor calculus and relativistic fields. Cambridge University Press, Cambridge (1984). 
[22] W H Press \& J M Bardeen. Non-conservation of the Newman-Penrose conserved quantities. Phys. Rev. Lett. 27, 1303 (1971).

[23] R K Sachs. Gravitational waves in general relativity VIII. Waves in asymptotically flat spacetime. Proc. Roy. Soc. Lond. A 270, 103 (1962).

[24] J Stewart. Advanced General Relativity. Cambridge University Press. U.K. 1993.

[25] P Székeres. The Gravitational Compass. J. Math. Phys. 6, 1387 (1965).

[26] J A Valiente Kroon. Conserved quantities for polyhomogeneous spacetimes. Class. Quantum Grav. 15, 2479 (1998).

[27] J A Valiente Kroon. A comment on the Outgoing Radiation Condition for the gravitational field and the Peeling Theorem. gr-qc 9811034. 